\title{
Using Kantian Ethics in Medical Ethics Education
}

\author{
Chase M. Donaldson ${ }^{1}$
}

Published online: 11 October 2017

(C) International Association of Medical Science Educators 2017

\begin{abstract}
Contemporary medicine has unique challenges that render principlism inadequate as a sole paradigm for medical ethics education. Shortcomings of this ethical system include the often contradictory nature of the principles, difficulty with integration and internalization of the principles, and the inadequate treatment of moral relativism. Principlism has also contributed to the rise of consumerism in medicine by making autonomy "chief among equals" of the principles. The ethics of Immanuel Kant is offered as a potential helpful addition to medical trainee ethics curricula. Kant's Categorical Imperative to "act in such a way that you treat humanity...never merely as a means to an end, but always at the same time as an end" respects patient autonomy and dignity while simultaneously offering an ethical foundation for the relationship between the patient and the physician. The concept of autonomy is discussed, specifically how Kant's idea of autonomy is very different from that in contemporary medicine, and is in fact more nuanced and helpful for the ethical development of physicians. By focusing on intentionality as opposed to outcomes, Kant's philosophy also is applicable to the "blame and shame" medical culture, establishes a deeper sense of duty for the physician, and is clinically helpful for patient and peer interaction.
\end{abstract}

Keywords Ethics · Graduate medical education · Principlism $\cdot$ Medical humanities $\cdot$ Trainee attitudes

Chase M. Donaldson

Chase.donaldson@gmail.com

1 Department of Pulmonary and Critical Care, William Beaumont Army Medical Center, Bldg 9, 10th Floor, 5005 N. Piedras, El Paso, TX 79920, USA
Traditionally, medical education has emphasized knowledge and procedural-based competencies while assuming the presence of the "softer" characteristics of the physician such as a developed ethical intuition and empathy. However, the nineteenth and twentieth centuries saw no shortage of systemic ethical and empathetic shortcomings which undermined the public trust in the physician-patient relationship. At the same time, American medicine has experienced a rapid paradigm shift to a medical system characterized by bureaucratic educational hierarchies, corporate-based medical practice, and the electronic medical record which have generally contributed to a dehumanization of the medical profession. There is currently a focus in medical education towards developing more empathetic and reflective physicians in order to correct these perceived historical deficiencies, and to give medical learners the tools to develop a professional identity to combat the systemic challenges in modern medicine that undermine the physicianpatient relationship $[1,2]$.

\section{Inadequacy of Principlism}

Contemporary medical ethics education centers on the Georgetown mantra of principlism, which is a method of ethical decision-making that highlights four principles or values that are said to have a universal, cross-cultural appeal. These principles - autonomy, beneficence, nonmaleficence, and justice - are invoked when examining an ethical dilemma, and an analysis ideally yields a solution that best balances the demands of each principle [3]. The ethical system has significant appeal in terms of its accessibility, its seemingly cross-cultural generalizability, flexibility, and potential for nuance through vigorous examination of the principles. Yet, from a pragmatic perspective, it suffers from a number of limitations that render 
it problematic as the crux upon which most of medical education relies $[4-8,14]$.

Because it is a system that calls for examining ethical issues from the perspectives of the four principles, the most common criticism is that the principles can be contradictory [9]. For example, alterations in mental status, psychiatric illness, intellectual disability, and even lack of education or emotional state can cause significant conflict between the principles of autonomy and beneficence or justice due to a patient's or surrogate's inability to process complex medical information. Solutions to this conflict have been exhaustively worked out in the bioethics literature over the past few decades, but they quickly become very technical, and therefore difficult to internalize and utilize in daily practice, particularly for physicians without prior exposure to, or experience with, medical ethics. In addition, what is being taught as principlism is in fact a significantly truncated version of the actual ethical system and has been described as "more akin to a checklist than an exposition of a nuanced moral framework [10]." Although principlism can be helpful for identifying the conflicts and nuances in potential ethical questions, its failure to consistently yield normative solutions to bedside problems and oversimplification in its presentation to medical learners renders sole reliance upon this ethical framework problematic for bedside care.

Beauchamp and Childress attempt to answer the criticism of contradictory principles by arguing that the principles must be balanced and that one principle can override another given that "adequate reasons are presented [11]." A set of criteria for doing this is provided, the primary one being that "better reasons can be offered to act on the overriding norm than on the infringed norm." Another attempt to salvage principlism is through the development of the concept of specification, whereby the principles are interpreted in the specific context (who, what, when, where, etc.) of the ethical dilemma, so as to clarify the norms and therefore reduce the conflict between the principles [12]. The implication is that if the ethical dilemma can be reduced to its morally relevant features through the particular context of the dilemma, somehow there will cease to be a conflict. Of course this is not true: moral conflict is inevitable and the framing of the specification process may be very different between two different people so as to confirm the moral intuition of the individual. Balancing has the same shortcoming. How to balance the principles ultimately will rely on subjective judgments tied to individual moral intuitions and social norms. There is no reasoned judgment for how to balance the principles, and thus there is no satisfactory objective normative function of the principles.

In addition to the issue of conflicting principles, another common criticism of principlism is the later clarification by Gillon that the principles are not actually equal in terms of values, but that autonomy is "first among equals [7, 13, 14]." If autonomy has indeed become the most important ethical value when considering ethical issues in medicine, it should be of no surprise that a medical culture traditionally informed by the ethos of "do no harm" and beneficence to the patient has become one that now seems to be chiefly informed by the unfettered and often uninformed will of the patient, at any medical cost. In practice, this perspective has given rise to what is now the era of "patient centered medicine" and its unfortunate derivatives - medical consumerism and patient satisfaction surveys - the effects of which have been deleterious to the patient-physician relationship and potentially to the health of patients themselves [15].

Other criticisms identify principlism as being overly reductive in its approach by narrowing the scope of focus on ethical inquiry to just the four principles, thus blinding the medical learner to other moral obligations in their medical practice such as making amends for medical errors or acknowledging and attempting to allay patient suffering [10]. Principlism has also proven inept at dealing with the problem of moral relativism [16].

\section{A Role for Kantian Ethics}

To address the challenges of medical ethics in medical education, a curriculum with a focus on comparative practical ethics, to include Kantian ethics, would better serve the needs of medical learners than curricula solely focused on principlism. A comparative practical ethics would involve approaching problems or scenarios in medical ethics from many different ethical approaches including principlism, virtue ethics, and utilitarianism. The potential contribution of Kantian ethics will be the focus of the remainder of this paper.

Kant's philosophy is generally placed under the category of deontological ethics. Deontology describes a philosophic approach where right and wrong is determined by the adherence to certain obligations or duties and is not dependent on outcomes, as in consequentialism. These duties, for Kant, originate apart from superstition, religion, tradition, and notably from empiricism as well, instead being derived from rational thought. Kant derived what he called the Categorical Imperative, that is, a moral obligation from which other obligations and duties derive. This moral law is universal and accessible to all humans.

Kant identifies rational thought as the unique characteristic of humans that separates man from beast, and this characteristic thereby accords an inherent dignity and worth to each human. Humans are said to act autonomously when they act in accordance with reason because when one utilizes reason, his or her humanity is asserted. For Kant then, humans are compelled to follow this universal law, the Categorical Imperative, by virtue of being human, and transgressing this law is a denial of that which makes one human [17]. 


\section{The Categorical Imperative and Autonomy}

Kant formulated the Categorical Imperative in three different ways, but its second formulation - act in such a way that you treat humanity, whether in your own person or in the person of any other, never merely as a means to an end, but always at the same time as an end - is the most relevant to our discussion of medical education. From Kant's philosophy, if every individual is a rational creature and therefore subject to the same universal moral law, each person possesses an inherent dignity, which ought to be respected by other rational creatures. This is an appealing proposition for contemporary medical ethics, because it personalizes the relationship with the patient by emphasizing a common humanity, despite the power differential in the relationship between physicians and patients. It satisfies the contemporary medical ethical zeitgeist by respecting the autonomy and dignity of the individual and offers a general grounding in the relationship between the patient and the physician by asserting a common bond of humanity between them. By establishing that the patient is an agent with free will, this reinforces the fact that, as Hippocrates wrote in his Oath, the purpose of the patient encounter is "for the benefit of the sick." For medical trainees, the Categorical Imperative thus mandates that all actions undertaken on behalf of the patient ought to be done by treating the patient as an end, which makes sense on both a theoretical and practical basis, and is applicable not only in ethical dilemmas, but also in terms of a general disposition towards the patient. Indeed, if the ethical system starts at a place that emphasizes a common humanity and expresses a duty towards the patient, the general outlook of the physician to the patient may be less susceptible to the depersonalizing influences of modern medicine. Unlike principlism, this concept is easily applicable at the bedside and has a less abstract human element that is easy to internalize for the medical learner. In today's graduate medical education environment where patient care consists more of documentation than patient interaction $[18,19]$, possibly contributing to the cynicism and dehumanization of patients seen in medical training centers [20-22], this paradigm could be of value.

Interestingly, Kant's ideas of autonomy and dignity have been traditionally used for the purposes of informed consent and for advocating for the role of personal preference of the patient in medicine. However, Kant's meaning of autonomy is very different from our current understanding [23]. For Kant, autonomy means an individual acting in accordance with reason, which, as discussed earlier, is universally accessible. Therefore, patients' preferences can be wrong if, because of emotion, false perceptions, or an inadequate understanding, their decision making is not in line with either objective information or their value systems. The implications for contemporary medical practice of this interpretation are significant. The current model has the relationship between physician and patient being an almost consumerist one, where the physician evaluates the patient and offers options among which the patient chooses with minimal intervention from the physician, so as to not obstruct the patient's autonomy. For Kant, on the other hand, the physician-patient relationship would be more akin to a physician as guide, the patient's Virgil in scaling the maze of the medical system and medical decision making, with the physician explaining different medical options and based on his or her understanding of the patient's values and perspective, would help the patient arrive at the most reasonable choice for that patient.

\section{Intentionality and Duty}

Unlike consequentialism, where the ends of a given action are given moral weight, for Kant, what is important for the individual is intentionality. Instead of focusing on the outcomes of a procedure, a particular patient interaction, or medical decision making, centering the ethical conflict on the physician's intention can be valuable. Medical care is often put in terms of outcomes, and this can translate into the ethical realm as well. If there is a bad outcome, there must be a bad doctor who did not do the right thing. Physicians can internalize this worldview, which in the inevitability of a bad outcome can be traumatic for the physician. Centering the good or bad of an ethical situation on the intentions of the physician prevents this maladaptive thought pattern. Establishing a focus upon intentionality during the early stages of medical education could potentially alleviate the current "blame and shame" culture of medical training. This is not to say that this concept of intentionality is the only approach to bad outcomes and medical errors, as a robust approach requires the synthesis of procedural skill, communication/teamwork, medical decision making, and systems-based practice. On the level of the individual physician, however, the anxieties surrounding the uncertainties of patient care would be well served by a culture that does not necessarily expect perfection in patient outcomes, but of excellence in caring and a focus on a disposition towards the good of the patient.

Conflicts of interest that arise in medicine are similarly well addressed by the paradigm of intentionality. The relationship of physicians to drug or device representatives, interpersonal conflicts between colleagues, and between physicians and their patients can all affect behavior, sometimes to the deleterious effects of patient care. In the moment when weighing the sometimes very different motivations in deciding how to act, or when competing obligations exist, the Kantian approach to primarily consider the intention of the physician in using patients as ends in themselves is appealing and easier to apply than an abstract consideration of disparate principles.

Duty is a concept of deontology that is fairly foreign to contemporary medicine, but has deep roots in Western philosophy and continues to have importance today in most non- 
Western cultures. The etymology of the term denotes the idea of a debt or an obligation owed to something or someone else. For Kant, duty means acting in accordance with the Categorical Imperative as an acknowledgement and affirmation of one's dignity as a rational creature. Extending this further for the physician, this means that the physician has a duty to treat his or her patients as an end and not merely as a means. The strength of the word duty over the words "should" or "ought" as we usually think about medical ethics reflects that there is actually an obligation a physician has towards his or her patient. This sentiment is unfortunately often absent from contemporary medical culture. This gives new meaning to the idea of physicians in training "taking ownership of their patients" and generally has the potential to change the disposition of the physician in training to viewing modern medicine, even with all of its discontents, as a much more solemn and sacred enterprise, changing what is a job into a vocation. A deeper, more internalized, almost transcendent importance of the role of the physician in honoring the dignity of the patient could alter the contemporary currents in medical culture that function to undermine, commercialize, and compromise the physician's engagement with the patient.

\section{Kant's Limitations and Moving Forward}

Utilizing Kant's philosophy for medical education is not a panacea. There is no perfect ethical theory that is philosophically rigorous, practical, and accessible to the physician. There are both practical and philosophical difficulties with Kant in medical ethics education. From a practical perspective, Kant's understanding of autonomy could be met with protests as it does open the door for a more paternalistic disposition towards the patient. The idea of intentionality, though helpful from the perspective of medical culture and as an introduction to medical learners, is also inadequate on a systemic level to evaluate ethical behavior. From a philosophical perspective, Kant can suffer from lack of accessibility because of his prolific writing and sometimes technical text. His first and probably most important formulation of the Categorical Imperative is dense and not even completely understood among ethicists in terms of how to put it into practice. There are also concerns regarding the cohesiveness of the three formulations. Finally, although concepts of dignity, autonomy, and not treating others as means to an end is emotionally satisfying, part of utilizing Kant's philosophy is understanding the rational methods he uses to generate the maxims that guide ethical behavior in accordance with reason, which can be difficult.

Kantian ethics, with an emphasis on the concepts of patient dignity, autonomy, and the second formulation of the Categorical Imperative, could be a helpful addition for medical ethics education. Though inadequate as a sole paradigm for the whole of medical ethics, with principlism, among others, still needing to serve a major role, utilizing Kant's Categorical Imperative in medical education as one of the ethical paradigms used to educate trainees about medical ethics could prove helpful to address the challenges unique to contemporary medicine. It offers a paradigm that places the subject of one's actions, the patient, as the end of all ethical action, and would therefore be easily integrated into the current culture of medical ethics that emphasizes patient-centered medicine. Finally, approaching medical ethics from a multimodal approach by utilizing comparative ethics provides an avenue for the medical trainee to become more involved and potentially interested in the richness and diversity of ethical theory itself. The more ethically literate physician would then be one who recognizes the origins of the diversity of opinions in medical ethics which would not only help the patient, but from a Kantian perspective, would compel the budding physician towards his or her imperfect duty of self-development, the sine qua non of education itself.

Acknowledgments The author would like to thank Dr. Robert J. Walter and Dr. Michael April for their assistance with draft preparation and technical editing.

\section{Compliance with Ethical Standards}

Conflicts of Interest The author declares that there is no conflict of interest.

Ethical Approval Not applicable

Disclaimer The expressed views are that of the author and do not necessarily represent the views of William Beaumont Army Medical Center, the Army, or the Department of Defense.

\section{Previous Presentation None}

\section{References}

1. Ng SL, Kinsella EA, Friesen F, et al. Reclaiming a theoretical orientation to reflection in medical education research: a critical narrative review. Med Educ. 2015 May;49(5):461-75.

2. Kelm Z, Womer J, Walter JK, et al. Interventions to cultivate physician empathy: a systematic review. BMC Med Educ. 2014 Oct 14;14:219.

3. Beauchamp TL, Childress J. Principles of biomedical ethics. 1st ed. New York: Oxford University Press; 1979.

4. Callahan D. Principlism and communitarianism. J Med Ethics. 2003;29:287-91.

5. Agledahl K, et al. Choice is not the issue. The misrepresentation of healthcare in bioethical discourse. J Med Ethics. 2011;37:212-5.

6. Tubbs J. Christian theology and medical ethics: four contemporary approaches. Norwell: Kluwer Academic Publishers; 1995. p. 2933.

7. O'Neill A. Autonomy and Trust in Bioethics (Gifford lectures). New York: Cambridge University Press; 2002.

8. Komrad MS. A defence of medical paternalism: maximizing patients' autonomy. J Med Ethics. 1983;9(1):38-44. 
9. Pellegrino E. The four principles and the doctor-patient relationship: the need for a better understanding. In: Gillon R, editor. Principles of Health Care Ethics. Chichester: John Wiley \& Sons; 1994. p. 353-66.

10. Fiester A. Viewpoint: why the clinical ethics we teach fails patients. Acad Med. 2007;82(7):684-9.

11. Beauchamp TL, Childress JF. Principles of biomedical ethics. 4th ed. New York: Oxford University Press; 1994.

12. Richardson HS. Specifying norms as a way to resolve concrete ethical problems. Philos Public Aff. 1990;19(Fall):279-310.

13. Gillon R. Ethics needs principles - four can encompass the restand respect for autonomy should be "first among equals". J Med Ethics. 2003;29:307-12.

14. Pellegrino ED, Thomasma DC. For the patient's good: the restoration of beneficence in health care. Oxford: Oxford University Press; 1988.

15. Fenton J, Jerant AF, Bertakis AD, et al. The cost of satisfaction: a national study of patient satisfaction, health care utilization, expenditures, and mortality. Arch Intern Med. 2012;172(5):405-11.

16. Dawson A, Garrard E. In defence of moral imperialism: four equal and universal prima facie principles. J Med Ethics. 2006;32:200-4.
17. Gregor M, et al. Critique of practical reason. New York: Cambridge University Press; 1997.

18. Block L, Habicht R, Wu A, et al. In the wake of the 2003 and 2011 duty hours regulations, how do internal medicine interns spend their time? JGIM. 2013;28(8):1042-7.

19. Oxentenko AS, West CP, Popkave C, et al. Time spent on clinical documentation: a survey of internal medicine residents and program directors. Arch Intern Med. 2010;170(4):377-80.

20. Hojat M, Vergare MJ, Maxwell K, et al. The devil is in the third year: a longitudinal study of erosion of empathy in medical school. Acad Med. 2009;84(9):1182-91.

21. Feudtner C, Christakis DA, Christakis NA. Do clinical clerks suffer ethical erosion? Students' perceptions of their ethical environment and personal development. Acad Med. 1994;69(8):670-9.

22. Hafferty FW, Franks R. The hidden curriculum, ethics teaching, and the structure of medical education. Acad Med. 1994;69(11):86171.

23. Seeker B. The appearance of Kant's deontology in contemporary Kantianism: concepts of patient autonomy in bioethics. J Med Philos. 1999;24:43-66. 